\title{
Diagrammatic Analysis of Subarachnoid Contrast Spread in the Contralateral Oblique View: A Technical Report
}

\author{
Julie Petro, MD, Ivan Urits, MD, Thomas Simopoulos, MD, Mark R. Jones, MD, and Jatinder Gill, MD
}

Interlaminar epidural steroid injections are commonly used in clinical practice for the relief of low back pain. Reliable deposition of medication in the epidural space is important in producing significant results. Pathological conditions, including vertebral osteoarthritis, degenerative disc disease, and spinal stenosis, are frequently treated with epidural steroid injections. Associated degenerative features such as ligamentum flavum hypertrophy, facet joint hypertrophy, and osteophyte formation can lead to significant alterations in the anatomy of the epidural space. Epidurograms, prior to steroid injection, aid the physician in identifying the epidural space, as a false loss of resistance can occur. Extradural, subdural, intradural, and subarachnoid contrast spread has been demonstrated in the anteroposterior and lateral views; but, has not previously been demonstrated in the contralateral oblique (CLO) view. This technical report is the first to demonstrate concomitant epidural and subarachnoid spread in the contralateral oblique view after inadvertent low volume subarachnoid injection, as might happen in clinical practice.
In this technical report, we demonstrate both epidural and subarachnoid contrast spread in the contralateral oblique view and compare it to the anteroposterior view.

In an oblique view, epidural contrast spread is observed to approximate the ventral margin of the superior aspect of the lamina while subarachnoid contrast spread has a distinct demarcation at the ventral laminar margin. In an anteroposterior view, epidural spread is confirmed by spread of the dye along the nerve roots and beyond the margins of the pedicles.

The CLO view provides an accurate and consistent visualization of the epidural space; irrespective of the obliquity, epidural contrast is observed to approximate the ventral margin of the superior aspect of the lamina. In the CLO view, if contrast spread does not approximate the lamina, then intrathecal injection must be considered.

Key words: Epidural steroid injection, epidurogram, epidural spread, subarachnoid injection, intrathecal spread, fluoroscopy, contralateral oblique view
Epidurograms are performed to confirm needle tip location in the epidural space and to demonstrate adequate spread of the therapeutic injectate to the suspected site of pain. It has been shown that inci-

From : Harvard Medical School, Beth Israel Deaconess Medical Center

Author for correspondence: Ivan Urits, MD

Address: Harvard Medical School, Beth Israel Deaconess Medical Center, 330 Brookline Ave, Boston, MA 02215

E-mail: iurits@bidmc.harvard.edu dence of false loss of resistance can be high, thus leading to non-epidural deposition of medication $(1,2)$. Similarly, accessing the target pathology has been shown to influence outcomes (3). Epidurograms help differentiate epidural spread from extradural, subdural, intradural, and subarachnoid spread, and have previously been described on anteroposterior, lateral, and contralateral oblique views (4-6). The pattern of subarachnoid contrast spread; however, has never been described in the contralateral oblique (CLO) view. We have previously proposed that epidural contrast spread approaches or touches the superior margin of the lamina in the contralateral oblique view 
(3). As such, if this pattern is not visualized, then the possibility of subarachnoid injection must be considered. Here we present a technical report that confirms what we have hypothesized.

\section{METHODS}

A 72-year-old male with low back pain and radicular symptoms underwent an attempted L4-5 interlaminar epidural steroid injection, complicated by dural puncture, as revealed by subarachnoid spread of the contrast. Subsequently an epidural steroid injection was successfully performed at the L5-S1 level. The anteroposterior and oblique images of concomitant subarachnoid and epidural contrast spread are presented below along with a diagrammatic analysis.

\section{RESULTS}

Figure $1(\mathrm{~A})$ shows subarachnoid spread at the L4-5 level. As expected, the contrast spread is confined within the medial margin of the pedicles and has a classic appearance of a myelogram. Figure 1(B) shows both intrathecal spread at the L4-5 level and epidural spread at the L5-S1 level. The epidural spread is confirmed by spread of the dye along the nerve roots and beyond the margins of the pedicles. Figure 1 (C) shows concomitant epidural and subarachnoid spread in the contralateral oblique view at 45 degrees. The contrast approaches the ventral margin of the lamina with epidural spread but remains distinctly separate from the ventral laminar margin when it is subarachnoid (Fig. 2).

\section{DISCUSSION}

We have previously presented the variability in epidural contrast spread patterns. The epidural space is non-homogenous in nature, with significant inter- and intra-individual variability. The space has distinct posterior, lateral, and anterior compartments. Various physiological and pathological conditions such as aging, spinal stenosis, disc displacement, and epidural scarring can further lead to significant alterations in the anatomy of the epidural space (4). When visualized in the oblique view, irrespective of the obliquity, the contrast is observed to approximate the ventral margin of the superior aspect of the lamina. The ligamentum flavum originates from the superior ventral margin of the lamina and is minimal

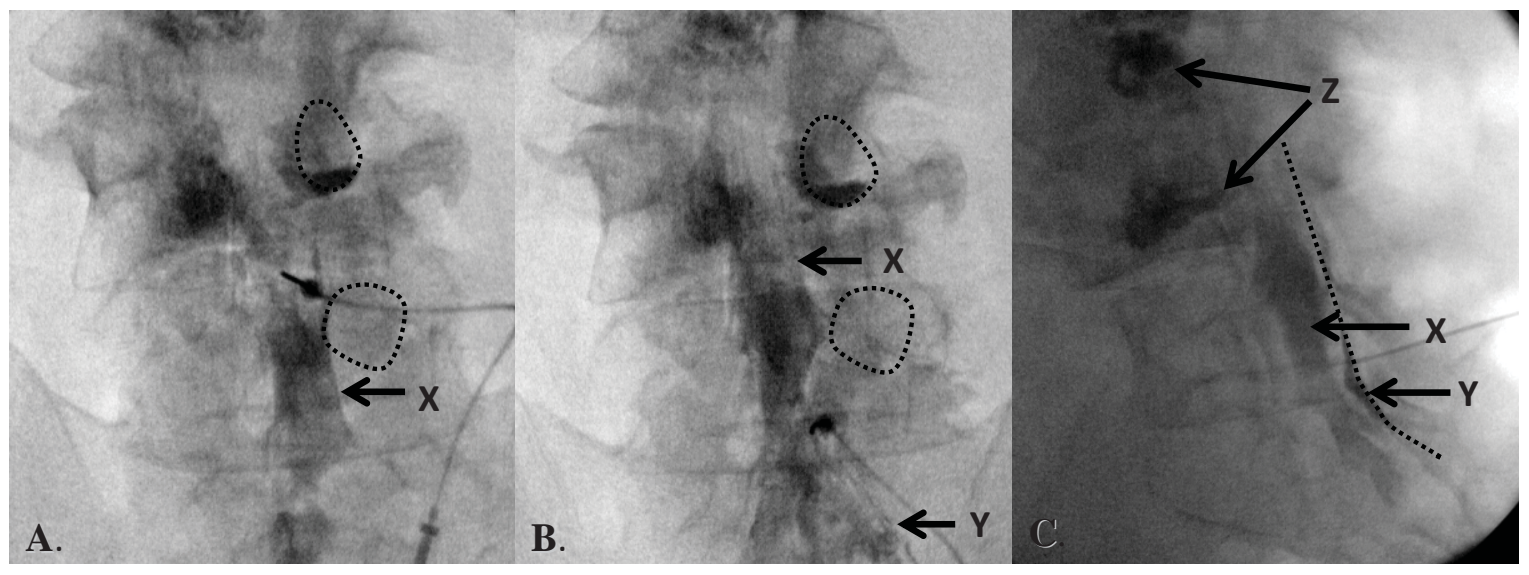

Fig. 1. A). Anteroposterior View (AP) view showing dural puncture at the L4-5 level demonstrated by intrathecal spread of contrast $(\mathrm{X})$ distinctly within the boundaries of the dura mater and medial margin of the pedicles (dashed circles). Contrast spread does not go beyond the pedicles. B). Demonstrates epidural injection at the L5-S1 level. Note that contrast spread (Y) exceeds the boundaries of the pedicles (dashed circles) whereas lingering intrathecal spread at L4-5 is represented by $(\mathrm{X}) . \mathrm{C})$. Contralateral oblique view at 45 degrees, epidural contrast (Y) can be seen approximating the ventral margin of the lamina, while intrathecal contrast $(\mathrm{X})$ does not and is separated by a radiolucent gap. Note kyphoplasty at L3 and L4 (Z). 

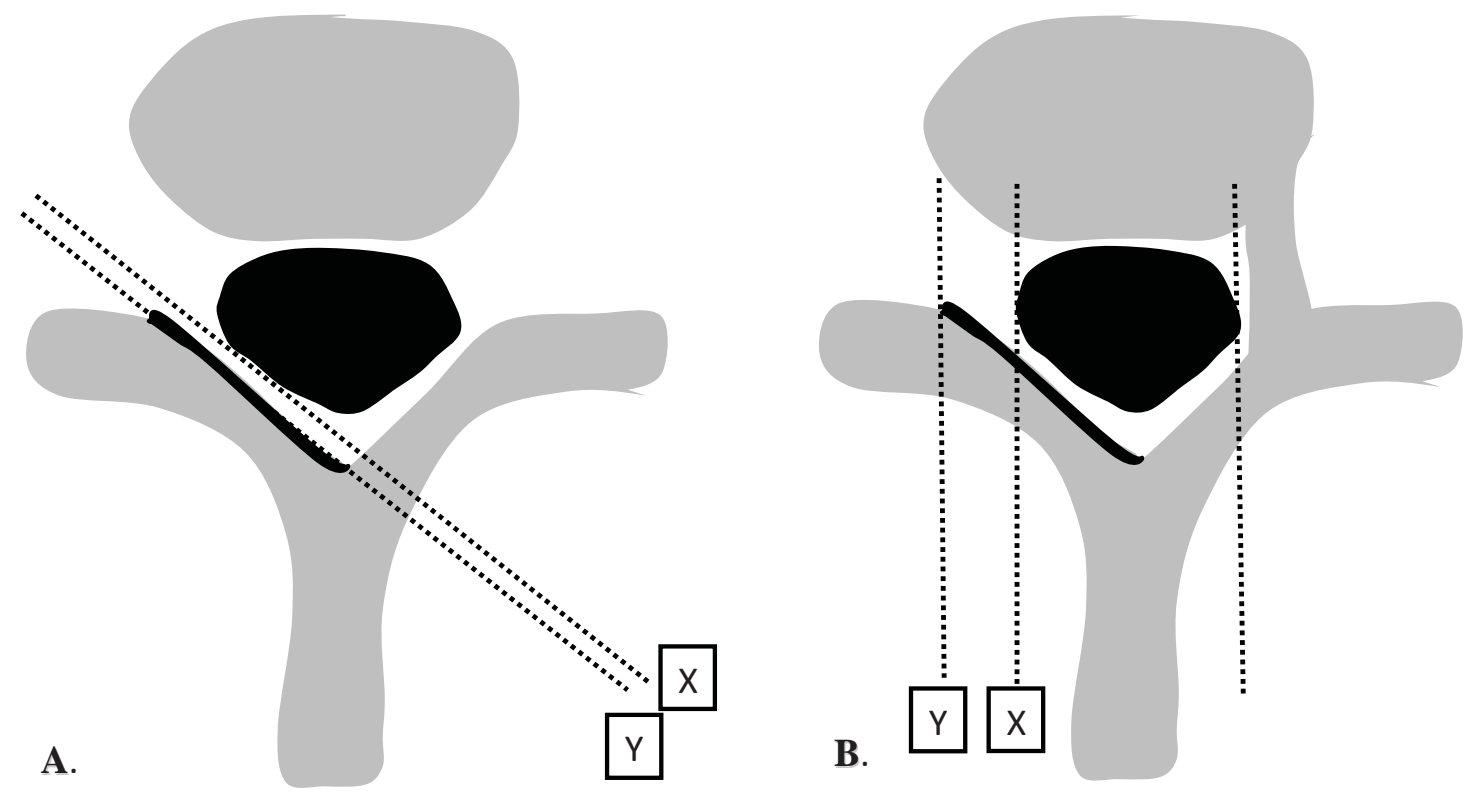

Fig. 2. A) Diagrammatic demonstration of why subarachnoid spread (X) remains separated from the ventral margin of the lamina by the epidural space. Epidural spread (Y) of contrast approximates the ventral margin of the superior aspect of the lamina. B) Diagrammatic representation for the basis of subarachnoid (X) spread remaining restricted to the medial margin of the pedicle.

in thickness at this locus; however, in the case of significant ligamentum hypertrophy, the needle may traverse beyond the ventral interlaminar line. This is a limitation of the CLO view, though it is predictable and corresponds to MRI findings (5). When contrast in the CLO view does not approximate the superior edge of the ventral laminar margin, subarachnoid spread should be suspected.

This technical report is the first to demonstrate concomitant epidural and subarachnoid spread in the contralateral oblique view after inadvertent low volume subarachnoid injection, as might happen in clinical practice. As we describe above, the contralateral oblique view establishes consistent radiographic landmarks which are useful to reliably differentiate epidural and intrathecal contrast spread. A larger prospective study of inadvertent subarachnoid injections may further validate this hypothesis.

\section{Acknowledgements and Conflict of Interest State- ment}

The authors acknowledge that there are no contributions or financial arrangements that represent a possible conflict of interest. The authors furthermore acknowledge that there was no technical help or contributions that do not justify authorship. Images in this report were created by the authors, and fluoroscopic images included have been de-identified. 


\section{REFERENCES}

1. White AH, Derby R, Wynne G. Epidural injections for the diagnosis and treatment of low-back pain. Spine 1980; 5:78-86.

2. Stojanovic MP, Vu T-N, Caneris O, Slezak J, Cohen SP, Sang $\mathrm{CN}$. The role of fluoroscopy in cervical epidural steroid injections. Spine 2002; 27:509-514.

3. Ghai B, Vadaje KS, Wig J, Dhillon MS. Lateral parasagittal versus midline interlaminar lumbar epidural steroid injection for management of low back pain with lumbosacral radicular pain. Anesthes Analg 2013; 117:219-227.

4. Gill J, Nagda J, Aner M, Simopoulos T. Cervical epidural con- trast spread patterns in fluoroscopic antero-posterior, lateral, and contralateral oblique view: A three-dimensional analysis. Pain Med 2017; 18:1027-1039.

5. Gill JS, Nagda JV, Aner MM, Keel JC, Simopoulos TT. Contralateral oblique view is superior to the lateral view for lumbar epidural access. Pain Med 2016;17:839-850.

6. Gill JS, Aner M, Jyotsna N, Keel JC, Simopoulos TT. Contralateral oblique view is superior to lateral view for interlaminar cervical and cervicothoracic epidural access. Pain Med 2015; 16:6880. 